\title{
Excess risk of fatal coronary heart disease associated with diabetes in men and women: meta-analysis of 37 prospective cohort studies
}

Rachel Huxley, Federica Barzi, Mark Woodward

\begin{abstract}
Objective To estimate the relative risk for fatal coronary heart disease associated with diabetes in men and women.

Design Meta-analysis of prospective cohort studies.

Data sources Studies published between 1966 and March 2005, identified through Embase and Medline, using a combined text word and MESH heading search strategy, in addition to studies from the Asia Pacific Cohort Studies Collaboration.

Review methods Studies were eligible if they had reported estimates of the relative risk for fatal coronary heart disease comparing men and women with and without diabetes. Studies were excluded if the estimates were not adjusted at least for age. Results 37 studies of type 2 diabetes and fatal coronary heart disease among a total of 447064 patients were identified. The rate of fatal coronary heart disease was higher in patients with diabetes than in those without $(5.4 v 1.6 \%)$. The overall summary relative risk for fatal coronary heart disease in patients with diabetes compared with no diabetes was significantly greater among women than it was among men: $3.50,95 \%$ confidence interval 2.70 to $4.53 v 2.06,1.81$ to 2.34 . After exclusion of the eight studies that had adjusted only for age, the difference in risk between the sexes was substantially reduced but still highly significant. The pooled ratio of the relative risks (women: men) from the 29 studies with multiple adjusted estimates was 1.46 (1.14 to 1.88 ).

Conclusions The relative risk for fatal coronary heart disease associated with diabetes is $50 \%$ higher in women than it is in men. This greater excess coronary risk may be explained by more adverse cardiovascular risk profiles among women with diabetes, combined with possible disparities in treatment that favour men.
\end{abstract}

\section{Introduction}

Type 2 diabetes has long been known as a risk factor for coronary heart disease and is conservatively estimated to increase the risk of a fatal event by twofold. ${ }^{2}$ The association between diabetes and coronary heart disease has been suggested to be stronger in women than in men, prompting the idea that diabetes eliminates, or substantially attenuates, the advantages of being female. ${ }^{3}$

Within the past decade three meta-analyses on this topic have produced conflicting results. ${ }^{4-6}$ Two concluded that women with diabetes were at increased risk of mortality from coronary heart disease compared with men, whereas the third found no difference. These discrepancies may have arisen from differences in the level of adjustment for other cardiovascular risk factors between included studies. For example, as lipid levels are directly affected by diabetes, and to differing degrees in men and women, ${ }^{7}$ overadjustment for lipid variables may attenuate any real difference between the sexes in relation to diabetes and fatal coronary heart disease. Adjusting for age alone, however, fails to take into account potential differences in the levels of other cardiovascular risk factors (for example, blood pressure, body mass index) between men and women with diabetes, which could generate a spurious difference between the sexes in the relative risk of mortality due to coronary heart disease associated with diabetes.

The Asia Pacific Cohort Studies Collaboration comprises a large number of prospective cohort studies in the region and was established to provide reliable evidence about the effects of a variety of putative factors on the risk of cardiovascular disease among populations in this region. ${ }^{8}$ We sought to produce a reliable and unbiased comparison of the relative risk for fatal coronary heart disease associated with diabetes separately for men and women by updating the earlier reviews with published data from the Asia Pacific Cohort Studies Collaboration as well as any cohort studies published before March 2005.

\section{Methods}

We identified relevant studies through Embase and Medline using a combined text word and MESH heading search strategy of the terms "diabetes", "gender", "sex", and "coronary heart disease". We included eligible studies from the three previous reviews and we also scanned references to identify any other relevant studies, as recommended by the meta-analysis of observational studies in epidemiology guidelines. ${ }^{9}$

\section{Data extraction}

We included prospective cohort studies if by March 2005 they had published quantitative estimates and standard errors (or confidence limits) of the relative risk for fatal coronary heart disease associated with diabetes for both men and women. Studies were excluded if they provided only an estimate of effect, with no means by which to calculate the standard error; if the estimates were not adjusted at least for age; or if the study population had been derived from patients with a history of cardiovascular disease. We also excluded studies if they were carried out among single sex populations thereby preventing any internal comparison of the effects of diabetes between the sexes. We did not assess the methodological criteria of the studies owing to the questionable merit of quality scoring in meta-analyses of observational studies. ${ }^{10}{ }^{11}$ Instead we investigated possible sources of heteroge-

Web references $w 1-w 46$, additional figures, and details of studies contributing data are on bmj.com 
neity by comparing the results for studies combined for particular characteristics (for example, method of diabetes diagnosis, country of origin). We examined the effect of duration of follow-up on estimates of effects by metaregression. ${ }^{12}$

\section{Data analysis}

We obtained summary estimates by means of a random effects approach using inverse variance weighting. ${ }^{12}$ Using the $\mathrm{I}^{2}$ statistic $(95 \% \mathrm{CI})$, we estimated the percentage of variability across studies attributable to heterogeneity rather than to chance variation. ${ }^{12}$ We estimated the women to men ratio of the relative risks for fatal coronary heart disease, comparing those with diabetes to those without, with $95 \%$ confidence intervals, both overall, for studies only with age adjusted estimates, and for studies only with multiple adjusted estimates. We assessed publication bias graphically using a funnel plot, plotting the natural $\log$ of the ratio of the relative risks against its standard error. We extracted differences in the mean (standard deviation) levels of systolic blood pressure, lipids, and body mass index in patients with and without diabetes from the Asia Pacific Cohort Studies Collaboration, weighted by their inverse variance and combined in a meta-analysis. All analyses were carried out using Stata version 8.

\section{Results}

Our search strategy yielded 5621 articles, of which 306 included primary data. Of these we excluded 234 articles either because there was no outcome of interest or because they reported duplicate data. A further 49 papers $^{2}{ }^{\text {w1-48 }}$ were excluded for various reasons (fig 1). The remaining 23 articles were eligible for inclusion in our review. These comprised 37 prospective cohort studies $^{8}{ }^{13-34}$ with information on 447064 patients $(45 \%$ women). Seventeen of these studies were included in the earlier reviews (table 1). The remaining 20 studies, 13 of which were derived from a previous publication from the Asia Pacific Cohort Studies Collaboration (see bmj.com), ${ }^{8}$ were identified for the purposes of this review (table 2). All but six of the 37 studies reported the number of patients with a diagnosis of diabetes at baseline (24 714, 31\% women). The duration of follow-up varied from between four to 36 years and the age range was between 15 and 98 years. Eleven of the 37 studies were from the United States, nine from Australia and New Zealand, nine from Asia, and eight from European countries.

\section{Coronary heart disease event rates}

In the 33 studies that reported the total number of deaths from coronary heart disease during follow-up, a total of 7570 of $420630(1.8 \%)$ people died. Twenty seven studies reported the number of fatal coronary heart disease events among participants by diabetes status (6335 of $333400,1.9 \%$ ); of these, 1203 ( $41 \%$ women) had diabetes. The rate of fatal coronary heart disease was substantially higher in people with diabetes than in those without $(5.4 \% v 1.6 \%)$. This difference was apparent in both sexes but more so among women (with and without diabetes $7.7 v 1.2 \%)$. The corresponding rates in men were $4.5 \%$ and $2.0 \%$.

\section{Summary estimate of relative risk for fatal coronary heart disease in patients with diabetes}

The overall summary estimate of the relative risk for fatal coronary heart disease associated with diabetes was significantly greater in women than it was in men (relative risk $3.50,95 \%$ confidence interval 2.70 to $4.53 v 2.06,1.81$ to $2.34 ; \mathrm{P}<0.0001$ : see figs $\mathrm{A}$ and $\mathrm{B}$ on bmj.com). We found significant heterogeneity

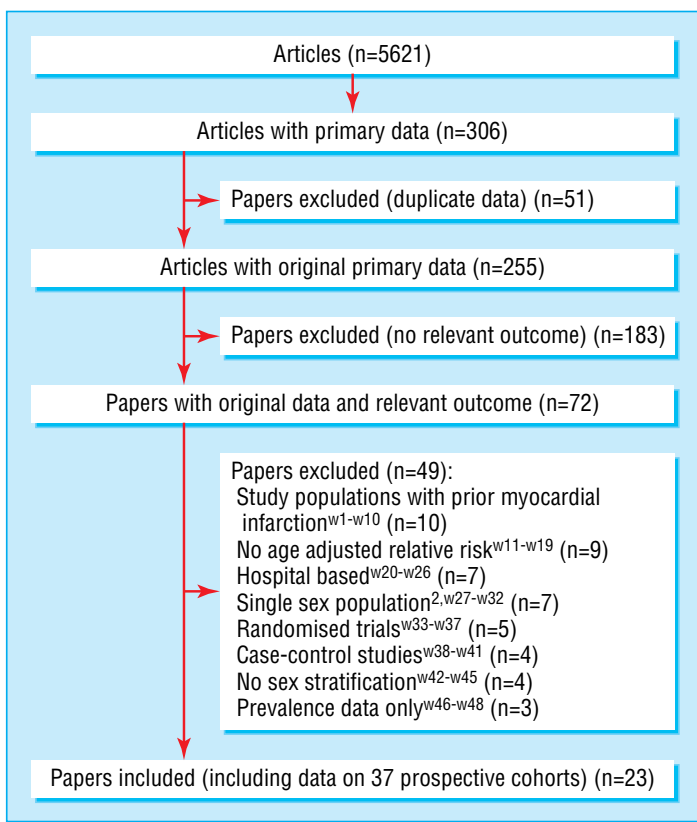

Fig 1 Flow chart of search strategy

across all studies (men: $\mathrm{I}^{2}=43 \%, 95 \%$ confidence interval $16 \%$ to $61 \%$; women: $74 \%, 65 \%$ to $81 \%$ ) that was substantially attenuated after exclusion of the eight studies with only age adjusted coefficients $(26 \%,-18 \%$ to $53 \%$ and $35 \%, 15 \%$ to $59 \%)$. Exclusion of these eight studies reduced the relative risk of fatal coronary heart disease in women with diabetes but not men $(2.95,2.39$ to $3.65 v 2.02,1.76$ to $2.31 ; \mathrm{P}=0.003$ for sex difference).

To further examine the effect of adjustment we considered the 22 studies that had provided both age and multiple adjusted coefficients (fig 2). All but two of these studies, in addition to adjusting for systolic blood pressure and total cholesterol, had adjusted for smoking. Adjustment resulted in a larger attenuation of the relative risk of fatal coronary heart disease among women than among men (fig 2). This greater attenuation in the relative risk among women with diabetes may be due in part to both the significantly higher levels of other cardiovascular risk factors compared with their male equivalents and the much larger difference in levels of risk factors between women with and without diabetes, compared with men with and without diabetes (table 3 ).

Ratio of relative risks for fatal coronary heart disease among men and women with diabetes

The pooled ratio of relative risks for diabetes from all 37 studies was 1.70 (1.27 to 2.27). Excluding the eight pairs of age adjusted relative risks, the ratio of relative risks was reduced to 1.46 (1.14 to 1.88). Thus the best evidence available suggests that the relative risk of fatal coronary heart disease associated with diabetes is about $50 \%$ higher in women than it is in men.

\section{Sensitivity analyses}

We carried out sensitivity analyses on the 29 studies for which multiple adjusted coefficients were available for both sexes (fig 3). We found no difference in the ratio of the relative risks for diabetes between men and women with diabetes according to fixed effects or random effects models, method of diabetes diagnosis (self report $v$ glucose measured), and region of study (Asia $v$ non-Asian countries). Metaregression indicated that the duration of study follow-up had no effect on the overall hazard ratios. Visual examination of the funnel plot showed no evidence 
Table 1 Characteristics of studies of coronary heart disease risk in individuals with and without diabetes that contributed to earlier reviews

\begin{tabular}{|c|c|c|c|c|c|c|}
\hline Reference & Cohort, country & Study size (No with diabetes) & $\begin{array}{l}\text { Age range } \\
\text { (years) }\end{array}$ & $\begin{array}{l}\text { Duration of study } \\
\text { (years) }\end{array}$ & $\begin{array}{l}\text { No of fatal coronary heart } \\
\text { disease events (No in } \\
\text { patients with diabetes) }\end{array}$ & $\begin{array}{l}\text { Variables adjusted for in } \\
\text { analyses }\end{array}$ \\
\hline Butler $1985^{13}$ & Tecumseh, United States & $\begin{array}{c}921 \text { men (43); } 937 \text { women } \\
(70)\end{array}$ & $>40$ & $12-20$ & 161 (19); 88 (17) & Age \\
\hline Pan $1986^{14}$ & $\begin{array}{l}\text { Chicago Heart Association } \\
\text { detection project in industry, } \\
\text { United States }\end{array}$ & $\begin{array}{c}11220 \text { men (377); } 8030 \\
\text { women (170) }\end{array}$ & $35-64$ & 9 & 286 (44); 47 (6) & $\begin{array}{l}\text { Age, smoking, systolic blood } \\
\text { pressure, total cholesterol, } \\
\text { electrocardiography, education }\end{array}$ \\
\hline Kleinman $1988^{15}$ & $\begin{array}{l}\text { National health and nutrition } \\
\text { examination survey I, United } \\
\text { States }\end{array}$ & $\begin{array}{c}3340 \text { men (189); } 4041 \text { women } \\
\text { (218) }\end{array}$ & $40-77$ & 9 & 321 (44); 160 (25) & $\begin{array}{l}\text { Age, smoking, systolic blood } \\
\text { pressure, total cholesterol, body } \\
\text { mass index }\end{array}$ \\
\hline Heyden $1990^{16}$ & Georgia, United States & 1284 men; 1420 women & $25-74$ & 4.5 & 524 & $\begin{array}{l}\text { Age, smoking, systolic blood } \\
\text { pressure, total cholesterol, } \\
\text { triglycerides, body mass index }\end{array}$ \\
\hline $\begin{array}{l}\text { Barrett-Connor } \\
1991^{17}\end{array}$ & $\begin{array}{l}\text { Rancho Bernardo California, } \\
\text { United States }\end{array}$ & $\begin{array}{c}1100 \text { men (207); } 1351 \text { women } \\
\text { (127) }\end{array}$ & $40-79$ & 14 & 127 (36); 77 (19) & $\begin{array}{l}\text { Age, smoking, systolic blood } \\
\text { pressure, total cholesterol, body } \\
\text { mass index, hormone replacement } \\
\text { therapy }\end{array}$ \\
\hline Fraser $1992^{18}$ & $\begin{array}{l}\text { California seventh day } \\
\text { adventists, United States }\end{array}$ & $\begin{array}{c}10376 \text { men (374); } 17282 \\
\text { women (812) }\end{array}$ & $>25$ & 6 & $136(13) ; 166(20)$ & $\begin{array}{l}\text { Age, smoking, hypertension, body } \\
\text { mass index, exercise }\end{array}$ \\
\hline Sievers $1992^{19}$ & Arizona, United States & $\begin{array}{c}2463 \text { men (536); } 2668 \text { women } \\
\text { (730) }\end{array}$ & $>15$ & 12.1 & $24(1) ; 12(0)$ & $\begin{array}{l}\text { Age, smoking, hypertension, } \\
\text { hypercholesterolaemia }\end{array}$ \\
\hline Seeman $1993^{20}$ & Connecticut, United States & $\begin{array}{c}1169 \text { men (156); } 1643 \text { women } \\
(230)\end{array}$ & $>65$ & 6 & 102 (18); $125(29)$ & $\begin{array}{l}\text { Age, smoking, systolic blood } \\
\text { pressure, body mass index }\end{array}$ \\
\hline Keil $1993^{21}$ & $\begin{array}{l}\text { Charleston heart (white } \\
\text { people), United States }\end{array}$ & 653 men, 741 women & $35-74$ & 30 & 237 & $\begin{array}{l}\text { Age, smoking, hypertension, } \\
\text { hypercholesterolaemia }\end{array}$ \\
\hline Keil $1993^{21}$ & $\begin{array}{l}\text { Charleston heart (black } \\
\text { people), United States }\end{array}$ & 333 men, 454 women & $35-74$ & 30 & 123 & $\begin{array}{l}\text { Age, smoking, systolic blood } \\
\text { pressure, total cholesterol, body } \\
\text { mass index, education }\end{array}$ \\
\hline Kannel $1995^{22}$ & $\begin{array}{l}\text { Framingham study, United } \\
\text { States }\end{array}$ & 5209 & $30-62$ & 36 & NA & Age \\
\hline Simons $1996^{23}$ & Dubbo, Australia & $\begin{array}{c}1155 \text { men (106); } 1472 \text { women } \\
\text { (101) }\end{array}$ & $>60$ & 5.2 & 223 (36); 200 (34) & Age \\
\hline Collins $1996^{24}$ & Fiji & $\begin{array}{c}472 \text { men (79); } 582 \text { women } \\
\text { (87) }\end{array}$ & $\geq 20$ & 11 & NA & $\begin{array}{l}\text { Age, smoking, hypertension, total } \\
\text { cholesterol }\end{array}$ \\
\hline Collins $1996^{24}$ & Fiji & $\begin{array}{c}605 \text { men (23); } 654 \text { women } \\
\text { (42) }\end{array}$ & - & 11 & NA & $\begin{array}{l}\text { Age, smoking, systolic blood } \\
\text { pressure, total cholesterol, body } \\
\text { mass index, survey area }\end{array}$ \\
\hline Folsom $1997^{25}$ & $\begin{array}{l}\text { Atherosclerosis risk in } \\
\text { communities study }\end{array}$ & 13446 & $45-64$ & $4-7$ & $\begin{array}{c}\text { Men, } 209 \text { (43); women, } 96 \\
\text { (33) }\end{array}$ & $\begin{array}{l}\text { Age, smoking, systolic blood } \\
\text { pressure, total cholesterol, high } \\
\text { density lipoprotein cholesterol, } \\
\text { triglycerides, body mass index }\end{array}$ \\
\hline Vilbergsson $1998^{26}$ & Reykjavik study, Iceland & $\begin{array}{c}9139 \text { men (267); } 9773 \text { women } \\
\text { (210) }\end{array}$ & $34-79$ & 17 & (92) & $\begin{array}{l}\text { Age, smoking, systolic blood } \\
\text { pressure, total cholesterol, } \\
\text { triglycerides, body mass index }\end{array}$ \\
\hline Jousilahti $1999^{27}$ & $\begin{array}{l}\text { Multinational monitoring of } \\
\text { trends and determinants in } \\
\text { cardiovascular disease, } \\
\text { Finland }\end{array}$ & $\begin{array}{c}7090 \text { men (262); } 7696 \text { women } \\
\text { (254) }\end{array}$ & $25-64$ & 7-12 & $231 ; 63$ & $\begin{array}{l}\text { Age, smoking, systolic blood } \\
\text { pressure, total cholesterol, high } \\
\text { density lipoprotein cholesterol, } \\
\text { body mass index }\end{array}$ \\
\hline
\end{tabular}

$N A=$ not available.

of publication bias (see fig C on bmj.com), which was confirmed by the Egger test.

\section{Discussion}

Diabetes poses a substantially greater increase in the risk of death from coronary heart disease among women than among men. Our finding is based on more than four times the amount of information available for previous reviews, making possible reliable quantitative estimates of the association between diabetes and risk for coronary heart disease between the sexes. Our findings are compatible with recent results from INTERHEART, a large case-control study of more than 15000 cases of acute myocardial infarction, which concluded that diabetes was a more significant coronary risk among women than it was among men (odds ratio $4.3,95 \%$ confidence interval 3.5 to 5.2 v $2.7,2.7$ to 3.0$){ }^{3 .}$

Several mechanisms could explain why diabetes has a greater adverse affect in women than in men. As supported by our analyses, diabetes may induce a more unfavourable cardiovascular risk profile among women. ${ }^{36}$ We found that women with diabetes not only have significantly higher levels of blood pressure and lipids than men with diabetes but that the difference in the levels among people with and without diabetes was significantly greater in women than it was in men. This would potentially explain why, after adjustment, the attenuation of the relative risk was considerably greater among women with diabetes than it was among their male equivalents, suggesting that the sex difference in coronary heart disease risk is mediated in large part by differences in the levels of cardiovascular risk factors.

Alternatively, the greater coronary risk associated with diabetes seen in women may reflect a treatment bias that favours men. Recent studies found that men with diabetes or established cardiovascular disease are more likely to receive aspirin, statins, or antihypertensive drugs than are women. For example, one study found that only $35 \%$ of women with diabetes or cardiovascular disease were prescribed a statin compared with $45 \%$ of men with similar medical histories. ${ }^{38}$ Similar findings were reported from the United Kingdom prospective diabetes study, ${ }^{39}$ where women with diabetes were significantly less likely to use aspirin compared with men. In two recent studies from the United 
Table 2 Characteristics of studies from Asia Pacific Cohort Studies Collaboration and other studies not included in previous reviews of coronary heart disease risk in people with and without diabetes

\begin{tabular}{|c|c|c|c|c|c|c|}
\hline Reference & Country & Study size (No with diabetes) & Age range (years) & $\begin{array}{l}\text { Duration of study } \\
\text { (years) }\end{array}$ & $\begin{array}{c}\text { No of fatal coronary heart } \\
\text { disease events (No with } \\
\text { diabetes) }\end{array}$ & Variables adjusted for in analyses \\
\hline Tanno $1977^{\star}$ & Japan & $\begin{array}{c}919 \text { men (62); } 1048 \text { women } \\
\text { (79) }\end{array}$ & $39-65$ & $16.4(15.3)$ & $18(2) ; 6(1)$ & $\begin{array}{l}\text { Age, smoking, systolic blood } \\
\text { pressure, total cholesterol }\end{array}$ \\
\hline Perth $1978^{*}$ & Australia & $\begin{array}{c}5123 \text { men (98); } 4599 \text { women } \\
\text { (95) }\end{array}$ & $20-90$ & $14.4(12.8)$ & 136 (10); 51 (3) & $\begin{array}{l}\text { Age, smoking, systolic blood } \\
\text { pressure, total cholesterol }\end{array}$ \\
\hline Singapore heart $1982^{\star}$ & Singapore & $\begin{array}{l}1168 \text { men (152); } 1123 \text { women } \\
\text { (113) }\end{array}$ & $20-89$ & $14.6(12.3)$ & $21(9) ; 8(2)$ & $\begin{array}{l}\text { Age, smoking, systolic blood } \\
\text { pressure, total cholesterol }\end{array}$ \\
\hline Akabane $1985^{\star}$ & Japan & $\begin{array}{c}812 \text { men (27); } 1020 \text { women } \\
\text { (18) }\end{array}$ & $40-69$ & $11.0(11.1)$ & $5(1) ; 2(1)$ & $\begin{array}{l}\text { Age, smoking, systolic blood } \\
\text { pressure, total cholesterol }\end{array}$ \\
\hline Newcastle $1988^{\star}$ & Australia & $\begin{array}{c}1713 \text { men (80); } 1690 \text { women } \\
\text { (39) }\end{array}$ & $21-77$ & $4.5(6.0)$ & $32(3) ; 10(1)$ & $\begin{array}{l}\text { Age, smoking, systolic blood } \\
\text { pressure, total cholesterol }\end{array}$ \\
\hline CVDFACTS $1988^{*}$ & Taiwan & $\begin{array}{c}2461 \text { men (77); } 3079 \text { women } \\
(67)\end{array}$ & $20-92$ & $6.0(6.4)$ & $6(1) ; 6(1)$ & $\begin{array}{l}\text { Age, smoking, systolic blood } \\
\text { pressure, total cholesterol }\end{array}$ \\
\hline ANHF $1989^{*}$ & Australia & $\begin{array}{c}4500 \text { men (96); } 4610 \text { women } \\
(66)\end{array}$ & $20-70$ & $8.3(8.2)$ & 58 (4); $19(1)$ & $\begin{array}{l}\text { Age, smoking, systolic blood } \\
\text { pressure, total cholesterol }\end{array}$ \\
\hline Melbourne 1990* & Australia & $\begin{array}{c}16905 \text { men (1206); } 24235 \\
\text { women (1015) }\end{array}$ & $27-75$ & $8.5(8.6)$ & 242 (38); 81 (13) & $\begin{array}{l}\text { Age, smoking, systolic blood } \\
\text { pressure, total cholesterol }\end{array}$ \\
\hline $\begin{array}{l}\text { Fletcher challenge } \\
1992^{\star}\end{array}$ & New Zealand & $\begin{array}{c}7369 \text { men (181); } 2856 \text { women } \\
\text { (84) }\end{array}$ & $20-89$ & $5.8(5.7)$ & 80 (13); $32(5)$ & $\begin{array}{l}\text { Age, smoking, systolic blood } \\
\text { pressure, total cholesterol }\end{array}$ \\
\hline KMIC 1992* & South Korea & $\begin{array}{c}106736 \text { men (10 736); } 53497 \\
\text { women (1513) }\end{array}$ & $35-59$ & $4.0(4.0)$ & 89 (19); 5 (1) & $\begin{array}{l}\text { Age, smoking, systolic blood } \\
\text { pressure, total cholesterol }\end{array}$ \\
\hline ALSA 1992* & Australia & 600 men (58); 554 women (27) & $65-98$ & $4.7(4.9)$ & $40(6) ; 20(1)$ & $\begin{array}{l}\text { Age, smoking, systolic blood } \\
\text { pressure, total cholesterol }\end{array}$ \\
\hline $\begin{array}{l}\text { Singapore NHS92 } \\
1992^{\star}\end{array}$ & Singapore & $\begin{array}{c}1593 \text { men (155); } 1710 \text { women } \\
\text { (165) }\end{array}$ & $20-70$ & $6.2(6.2)$ & 17 (8); 5 (3) & $\begin{array}{l}\text { Age, smoking, systolic blood } \\
\text { pressure, total cholesterol }\end{array}$ \\
\hline Kuusisto $1994^{28}$ & Finland & $\begin{array}{c}470 \text { men (74); } 828 \text { women } \\
\text { (155) }\end{array}$ & $65-74$ & 3.5 & $75(39)$ & Age \\
\hline Laakso $1995^{29}$ & Finland & $\begin{array}{c}1219 \text { men (581); } 1213 \text { women } \\
\text { (478) }\end{array}$ & $45-64$ & 7.2 & 122 (97); 63 (61) & Age \\
\hline Qvist $1996^{30}$ & Sweden & $\begin{array}{c}2546 \text { men (96); } 2760 \text { women } \\
\text { (75) }\end{array}$ & $45-74$ & 10 & 189 (20); 75 women (18) & Age \\
\hline Busselton 1996* & Australia & $\begin{array}{c}2709 \text { men (109); } 2948 \text { women } \\
\text { (89) }\end{array}$ & $20-94$ & $20.5(20.2)$ & $306(14) ; 234(16)$ & $\begin{array}{l}\text { Age, smoking, systolic blood } \\
\text { pressure, total cholesterol }\end{array}$ \\
\hline Tunstall-Pedoe $1997^{31}$ & Scotland & $\begin{array}{c}5754 \text { men (86); } 5875 \text { women } \\
\text { (88) }\end{array}$ & $40-59$ & $6-9$ & 404 (NA); 177 (NA) & Age \\
\hline Nilsson $1998^{32}$ & Sweden & $\begin{array}{c}18825 \text { men (420); } 19454 \\
\text { women (356) }\end{array}$ & $25-74$ & 16 & $\begin{array}{c}\text { Men, } 1050 \text { (94); women, } 470 \\
\text { (87) }\end{array}$ & Age \\
\hline Imazu $2002^{33}$ & Japan & 400 men (78); 517 women (91) & 60 & $10-18$ & $29(12) ; 14(9)$ & $\begin{array}{l}\text { Age, smoking, hypertension, total } \\
\text { cholesterol, triglycerides, body } \\
\text { mass index, uric acid }\end{array}$ \\
\hline Juutilainen $2004^{34}$ & Finland & $\begin{array}{c}1012 \text { men (429); } 1119 \text { women } \\
\text { (406) }\end{array}$ & $45-64$ & 13 & $\begin{array}{c}\text { Men, } 138 \text { (101); women, } 96 \\
(90)\end{array}$ & $\begin{array}{l}\text { Age, smoking, total cholesterol, } \\
\text { high density lipoprotein cholesterol, } \\
\text { triglycerides, body mass index }\end{array}$ \\
\hline
\end{tabular}

$\mathrm{NA}=$ not available.

${ }^{\star}$ Cohort study from Asia Pacific Cohort Studies Collaboration'; see bmj.com.

States, women with diabetes were also less likely to be treated with aspirin and lipid lowering agents or to achieve recommended levels of blood pressure or low density lipoprotein cholesterol than were men. ${ }^{41}$ Therefore more aggressive treatment of risk factors for coronary heart disease in

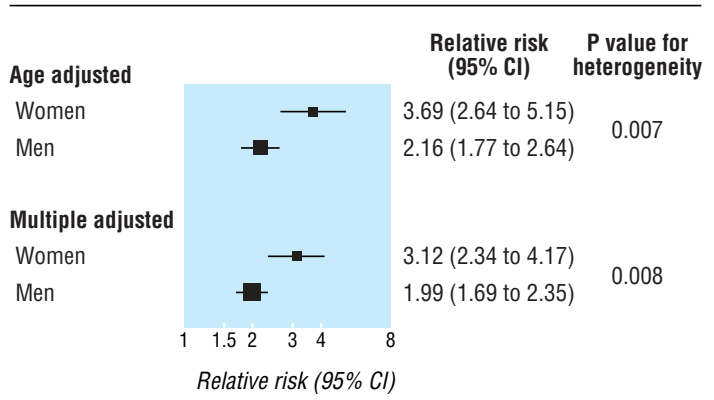

Fig 2 Overall summary estimates of relative risks and 95\% confidence intervals for fatal coronary heart disease in men and women with and without diabetes in 22 studies that reported both age and multiple adjusted coefficients men with diabetes may explain a large component of the excess risk associated with diabetes in women.

\section{Limitations of study}

As with previous reviews, the lack of individual patient data precluded further exploration of the effect of adjustment as well as the role of treatment differences on the association of diabetes with coronary risk among men and women. Moreover, informa-

Table 3 Mean baseline risk factor levels and differences among men and women with and without diabetes

\begin{tabular}{|c|c|c|}
\hline \multirow{2}{*}{ Risk factor } & \multicolumn{2}{|c|}{ Difference (diabetes-no diabetes) (95\% CI) } \\
\hline & Men* & Women* \\
\hline Systolic blood pressure (mm Hg) & 7.8 (7.5 to 8.1$)$ & 12.5 (12.0 to 13.0$)$ \\
\hline Total cholesterol (mmol/l) & $0.24(0.22$ to 0.26$)$ & $0.46(0.43$ to 0.49$)$ \\
\hline Triglycerides† (mmol/l) & 1.53 (1.41 to 1.66$)$ & 2.01 (1.88 to 2.14$)$ \\
\hline $\begin{array}{l}\text { High density lipoprotein cholesterol } \\
(\mathrm{mmol} / \mathrm{l})\end{array}$ & $-0.076(-0.1$ to -0.05$)$ & $-0.13(-0.16$ to -0.1$)$ \\
\hline Body mass index $\left(\mathrm{kg} / \mathrm{m}^{2}\right)$ & 0.69 (0.65 to 0.74$)$ & 1.98 (1.87 to 2.09 ) \\
\hline
\end{tabular}

*Data from Asia Pacific Cohort Studies Collaboration. ${ }^{8}$

†Log transformed before analysis and subsequently transformed back. 


\begin{tabular}{|c|c|c|c|c|}
\hline \multicolumn{3}{|l|}{ Statistical method } & $\begin{array}{c}\mathrm{P} \text { value for } \\
\text { heterogeneity }\end{array}$ & \multirow[t]{2}{*}{$\begin{array}{l}\text { P value for } \\
\text { heterogeneity } \\
\text { of sex } \\
\text { interaction }\end{array}$} \\
\hline \multirow{2}{*}{ Fixed effects } & Women & $=$ & & \\
\hline & Men & - & 0.001 & \multirow{3}{*}{0.70} \\
\hline \multirow[t]{2}{*}{ Random effects } & Women & $\rightarrow$ & \multirow{2}{*}{0.003} & \\
\hline & Men & $\leftarrow$ & & \\
\hline \multicolumn{5}{|c|}{ Method of diagnosis } \\
\hline \multirow[t]{2}{*}{ Self-report diabetes } & Women & $\rightarrow$ & \multirow{2}{*}{0.11} & \\
\hline & Men & $\rightarrow$ & & 0.45 \\
\hline \multirow[t]{2}{*}{ Glucose measured } & Women & 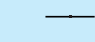 & \multirow{2}{*}{0.017} & \\
\hline & Men & - & & \\
\hline \multicolumn{5}{|l|}{ Region } \\
\hline \multirow[t]{2}{*}{ Asia } & Women & & \multirow{2}{*}{0.016} & \\
\hline & Men & $\rightarrow$ & & 0.17 \\
\hline \multirow{3}{*}{ Non-Asian countries } & $\begin{array}{l}\text { Women } \\
\text { Men }\end{array}$ & - & 0.027 & \\
\hline & & 1.5234 & 8 & \\
\hline & & e risk (95 & $\%$ Cl) & \\
\hline
\end{tabular}

Fig 3 Sensitivity analyses on basis of statistical method, method of diagnosis, and region

tion on menopausal status and hormone replacement therapy use was not available for the present analyses, therefore we could not exclude their potential confounding effect. Finally, as we did not have information on the duration of diabetes we were unable to confirm a previous study's finding that there is a difference between the sexes in the effect of duration of diabetes on fatal coronary heart disease. ${ }^{42}$

\section{Conclusion}

The excess risk of coronary death associated with diabetes is substantially higher in women than it is in men. This may be a consequence of diabetes inducing a more adverse cardiovascular risk profile in women, combined with a reduced likelihood of women receiving standard treatment and attaining recommended levels of other coronary heart disease risk factors. More aggressive treatment and better control of other coronary heart disease risk factor levels in women with diabetes is likely to substantially reduce the excess coronary heart disease mortality seen in this subgroup.

Contributors: All authors contributed to the interpretation of the analyses and drafting the manuscript. RH is guarantor.

Funding: RH is supported by a University of Sydney Sesqui postdoctoral fellowship. This work has received funds from the Australian National Health and Medical Research Council and from an unconditional educational grant from Pfizer.

Competing interests: None declared.

Ethical approval: Not required.

\section{What is already known on this topic}

People with type 2 diabetes are at a much greater risk of fatal coronary heart disease than those without diabetes

It is unclear whether the adverse effects of diabetes are greater for women than they are for men

\section{What this study adds}

The risk of death from coronary heart disease associated with type 2 diabetes is about $50 \%$ greater in women than it is in men
1 Kannel WB, McGee DL. Diabetes and cardiovascular disease: the Framingham study. JAMA 1979;241:2035-8.

2 Fuller JH, Shipley MJ, Rose G, Jarrett RJ, Keen H. Mortality from coronary heart disease and stroke in relation to degree of glycaemia: the Whitehall study. BMJ 1983;287:867-70.

3 Castell WP. Cardiovascular disease in women. Am J Obstet Gynecol 1988;158:1153-60.

4 Orchard TJ. The impact of gender and general risk factors on the occurrence of atherosclerotic vascular disease in non-insulin-dependent diabetes mellitus. Ann Med 1996;28:323-33.

5 Lee WL, Cheung AM, Cape D, Zinman B. Impact of diabetes on coronary artery disease in women and men: a meta-analysis of prospective studies. Diabetes Care 2000;23:962-8.

6 Kanaya AM, Grady D, Barrett-Connor E. Explaining the sex difference in coronary heart disease mortality among patients with type 2 diabetes mellitus. Arch Intern Med 2002:1737-45.

7 Walden CE, Knopp RH, Wahl PW. Sex differences in the effect of diabetes mellitus on lipoprotein triglyceride and cholesterol concentrations. N Engl J Med 1984;311:953-9.

8 Asia Pacific Cohort Studies Collaboration. The effects of diabetes on the risks of major cardiovascular diseases and death in the Asia-Pacific region. The Asia Pacific Cohort cardiovascular diseases and death in the Asia-Pacif
Studies Collaboration. Diabetes Care 2003;26:360-6.

9 Stroup DF, Berlin JA, Morton SC, Olkin I, Williamson GD, Rennie D, et al. Meta-analysis of observational studies in epidemiology: a proposal for reporting. Metaanalysis of observational studies in epidemiology (MOOSE) group. JAMA 2000;283:2008-12.

10 Greenland S. Invited commentary: a critical look at some popular meta-analytic methods. Am J Epidemiol 1994;140:290-6.

11 Juni P, Witschi A, Bloch R, Egger M. The hazards of scoring the quality of clinical trials for meta-analysis. JAMA 1999;282:1054-60.

12 Woodward M. Epidemiology: study design and data analysis. 2nd edn. Boca Raton: Chapman and Hall/CRC, 2005.

13 Butler WJ, Ostrander LD Jr, Carman WJ, Lamphiear DE. Mortality from coronary heart disease in the Tecumseh study: long-term effect of diabetes mellitus, glucose tolerance and other risk factors. Am J Epidemiol 1985;121:541-7.

14 Pan WH, Cedres LB, Liu K, Dyer A, Schoenberger JA, Shekelle RB, et al. Relationship of clinical diabetes and asymptomatic hyperglycemia to risk of coronary heart disease mortality in men and women. Am J Epidemiol 1986;123:504-16.

15 Kleinman JC, Donahue RP, Harris MI, Finucane FF, Madans JH, Brock DB. Mortality among diabetics in a national sample. Am J Epidemiol 1988;128:389-401.

16 Heyden S, Heiss G, Bartel AG, Hames CG. Sex differences in coronary mortality among diabetics in Evans County, Georgia.J Chronic Dis 1980;33:265-73.

17 Barrett-Connor EL, Cohn BA, Wingard DL, Edelstein SL. Why is diabetes mellitus a stronger risk factor for fatal ischaemic heart disease in women than in men. The Rancho Bernardo study. JAMA 1991;265:627-31.

18 Fraser GE, Strahan TM, Sabate J, Beeson WL, Kissenger D. Effects of traditional coronary risk factors on rates of incident coronary events in a low-risk population: the Adventist health study. Circulation 1992;86:406-13.

19 Sievers ML, Nelson RG, Knowler WC, Bennett PH. Impact of NIDDM on mortality and causes of death in Pima Indians. Diabetes Care 1992;15:1541-9.

20 Seeman T, Mendes de Leon C, Berkman L, Ostfeld A. Risk factors for coronary heart disease among older men and women: a prospective study of community-dwelling elderly. Am J Epidemiol 1993;138:1037-49.

21 Keil JE, Sutherland SE, Knapp RG, Lackland DT, Gazes PC, Tyroler HA. Mortality rates and risk factors for coronary disease in black as compared with white men and women. N Engl J Med 1993;329:73-8.

22 Kannel WB, Wilson PWF. Risk factors that attenuate the female coronary disease advantage. Arch Intern Med 1995;155:57-61.

23 Simons LA, McCallum J, Friedlander Y, Simons J. Diabetes, mortality and coronary heart disease in the prospective Dubbo study of Australian elderly. Aust NZ J Med 1996;26:66-74.

24 Collins VR, Dowse GK, Ram P, Cabealawa S, Zimmet PZ. Non-insulin-dependent diabetes and 11-year mortality in Asian Indian and Melanesian Fijians. Diabet Med 1996;13:125-32.

25 Folsom AR, Szklo M, Stevens J, Liao F, Smith R, Eckfeldt JH. A prospective study of coronary heart disease in relation to fasting insulin, glucose, and diabetes: the atherosclerosis risk in communities (ARIC) study. Diabetes Care 1997;20:935-42.

26 Vilbergsson S, Sigurdsson G, Sigvaldason H, Sigfusson N. Coronary heart disease mortality amongst non-insulin-dependent diabetic subjects in Iceland: the independent effect of diabetes: the Reykjavik study 17-year follow up. J Intern Med 1998;244:309-16.

27 Jousilahti P, Vartiainen E, Tuomilehto J, Puska P. Sex, age, cardiovascular risk factors, and coronary heart disease: a prospective follow-up study of 14786 middle-aged men and coronary heart disease: a prospective follow-up stid
and women in Finland. Circulation 1999;99:1165-72.

28 Kuusisto J, Mykkanen L, Pyorala K, Laakso M. NIDDM and its metabolic control predict coronary heart disease in elderly subjects. Diabetes 1994;43:960-7.

29 Laakso M, Ronnemaa T, Lehto S, Puukka P, Kallio V, Pyorala K. Does NIDDM increase the risk for coronary heart disease similarly in both low- and high-risk populations? Diabetologia 1995;38:487-93.

30 Qvist J, Johansson SE, Johansson LM. Multivariate analyses of mortality from coronary heart disease due to biological and behavioural factors. Scand J Soc Med 1996;24:67-76.

31 Tunstall-Pedoe H, Woodward M, Tavendale R, A'Brook R, McCluskey MK. Comparison of the prediction by 27 different factors of coronary heart disease and death in men and women of the Scottish heart health study: cohort study. BMJ 1997;315:722-9.

32 Nilsson PM, Johansson S-E, Sundquist J. Low educational status is a risk factor for mortality among diabetic people. Diab Med 1998;15:213-9.

33 Imazu M, Sumii K, Yamamoto H, Toyofuku M, Tadehara F, Okubo M, et al. Influence of type 2 diabetes mellitus on cardiovascular disease mortality: findings from the HawaiiLos Angeles-Hiroshima study. Diabetes Res Clin Prac 2002;57:61-9.

34 Juutilainen A, Kortelainen S, Lehto S, Ronnemaa T, Pyorala K, Laakso M. Gender difference in the impact of type 2 diabetes on coronary heart disease risk. Diabetes Care 2004:27:2898-904

35 Yusuf S, Hawken S, Ounpuu S, dans T, Avezum A, Lanas F, et al. Effect of potentially modifiable risk factors associated with myocardial infarction in 52 countries (the INTERHEART study): case-control study. Lancet 2004;364:937-52. 


\section{Research}

36 Wingard DL, Barrett-Connor EL, Ferrara A. Is insulin really a heart disease risk factor. Diabetes Care 1995;18:1299-304.

37 Fuller JH, Keen H, Jarrett RJ, Omer T, Meade TW, Chakrabarti R, et al. Haemostatic variables associated with diabetes and its complications. BMJ 1979;2:964-6.

38 Tonstad S, Rosvold EO, Furu K, Skurtveit S. Undertreatment and overtreatment with statins: the Oslo Health Study 2000-2001.J Intern Med 2004;255:494-502.

39 Cull CA, Neil HA, Holman RR. Changing aspirin use in patients with type 2 diabetes in the UKPDS. Diab Med 2004;21:1368-71.

40 Wexler DJ, Grant RW, Meigs JB, Nathan DM, Cagliero E. Sex disparities in treatment of cardiac risk factors in patients with type 2 diabetes. Diabetes Care 2005;28:514-20.

11 Persell SD, Baker DW. Aspirin use among adults with diabetes. Arch Intern Med 2004;164:2492-9.

42 Natarajan S, Liao Y, Sinha D, Cao G, McGee D, Lispsitz SR. Sex differences in the effect of diabetes duration on coronary heart disease mortality. Arch Intern Med 2005; $165: 430-5$
(Accepted 8 November 2005)

doi $10.1136 /$ bmj.38678.389583.7C

George Institute for International Health, University of Sydney, PO Box M201, Sydney, NSW 2050, Australia

Rachel Huxley senior epidemiologist

Federica Barzi senior research fellow

Mark Woodward professor of biostatistics

Correspondence to: R Huxley rhuxley@thegeorgeinstitute.org 